\title{
Urinary Retention and the Role of Indwelling Catheterization Following Total Knee Arthroplasty
}

\author{
P. Kumar, K. Mannan, A.M. Chowdhury, K.C. Kong, J. Pati \\ Department of Urology and Department of Orthopaedics, Homerton University Hospital, London, United \\ Kingdom
}

\begin{abstract}
Introduction: We aimed to investigate the rate of urinary retention after knee arthroplasty, the various factors involved in predicting those at risk for retention and to assess the impact of retention and catheterization on joint sepsis.

Materials and Methods: A retrospective review was conducted of all available case notes of patients undergoing total knee arthroplasty in a consecutive 2-year period (2000-2002). Adequate data was available for 142 patients.

Results: 142 patients underwent total knee arthroplasty. 19 patients were catheterized preoperatively for monitoring urine output. 123 patients were not catheterized. Urinary retention occurred in $19.7 \%$ (28/142). The mean day of catheterization for retention was 0.66 . The mean duration of catheterization in patients developing retention was 3.58 days and was 3 days in the patients catheterized pre - or perioperatively. Deep joint sepsis occurred in $2.1 \%$ (3/142) - only one had been catheterized and that was preoperatively. No case of infection had urinary retention or had a symptomatic urinary tract infection. The only factors predicting those at significant risk of retention following knee arthroplasty was a past medical history of urinary retention $(\mathrm{p}=0.049)$ and postoperative morphine requirement $(\mathrm{p}=0.035)$. No patients required urological surgical intervention at mean follow up of 1.97 years.

Conclusions: This study supports the use of indwelling urinary catheterization for patients developing urinary retention after total knee arthroplasty.
\end{abstract}

Key words: urinary retention; urinary catheterization; arthroplasty, replacement, knee; sepsis; urinary tract infection Int Braz J Urol. 2006; 32: 31-4

\section{INTRODUCTION}

Acute urinary retention is a common complication following total joint arthroplasty with the incidence ranging from 10.7 (1) to 84\% (2). This complication may rarely but significantly lead to deep joint sepsis (3). There is little in the literature however looking specifically at knee arthroplasty in this setting (4). This is surprising since joint sepsis is more common in knee versus hip arthroplasty (5) and when there is infection associated with a knee prosthesis this is more difficult to treat as com- pared with hip prostheses (6). It is known that bladder overdistension leads to an alteration in bladder function and there may be a delay in return to normal bladder physiology after catheter removal. A large residual itself also predisposes to urinary tract infection and bladder drainage in this situation prevents this. Therefore, it would be helpful to be able to predict those patients at risk of urinary retention postoperatively and observe them closely to catheterize either preventatively or early to prevent overdistension with its attendant complications. We therefore aimed to investigate the rate of urinary re- 
tention after knee arthroplasty, the various factors involved in predicting those patients at risk for retention and to assess the impact of urinary retention and catheterization on joint sepsis.

\section{MATERIALS AND METHODS}

A retrospective review was conducted of all available case notes of patients undergoing total knee arthroplasty in a consecutive 2-year period (20002002). One hundred and forty-two patients underwent total knee arthroplasty in this period and adequate data was available on all 142 patients. Variables noted included age, sex, rate of urinary retention, catheterization, urinalysis, urinary tract infection, joint sepsis, anesthetic type, use of patient controlled analgesia, postoperative morphine requirement, alpha blockade, past medical and urological history.

All patients received a $1.5 \mathrm{~g}$ dose of cefuroxime intravenously at induction and 2 further doses of 750 mg 8 and 16 hours postoperatively. If catheterization was subsequently required then $80 \mathrm{mg}$ gentamicin intramuscularly was administered prophylactically prior to insertion of the catheter. Similarly, before removing a catheter $80 \mathrm{mg}$ gentamicin was given intramuscularly. The results were tabulated and analyzed using the programs Microsoft Excel ${ }^{\mathrm{TM}}$ and Stats Direct ${ }^{\mathrm{TM}}$.

\section{RESULTS}

One hundred and forty-two patients underwent total knee arthroplasty. Nineteen patients were catheterised preoperatively for the monitoring of urine output. This left 123 patients of whom 28 were catheterised due to urinary retention postoperatively. Therefore $66.9 \%$ (95/142) patients in our study did not require catheterisation at all. Preoperative urinalysis was available for $66.2 \%$ (94/ 142) of patients. These results were universally negative. Patients were catheterised if they could not void postoperatively and were in discomfort or had a palpable bladder. Urinary retention occurred in $21.1 \%$ (30/142) this includes 2 patients with a preoperative catheter developed retention after its removal (Table-
1). The mean day of catheterisation for retention was 0.66 . The mean duration of catheterisation in patients developing retention was 3.58 days and was 3 days in the patients catheterised pre- or perioperatively. Deep joint sepsis occurred in $2.1 \%$ (3/142) - only one had been catheterised and that was preoperatively. No case of prosthetic infection occurred following post-operative urinary retention. No patients had a symptomatic postoperative urinary tract infection. The risk of developing acute urinary retention after knee arthroplasty did not correlate significantly with age, sex, past medical history, alpha blockade, anaesthetic type or use of patient controlled analgesia. There were two factors predicting those at significant risk of retention following knee arthroplasty. The first variable was a past medical history of urinary retention ( $p=0.049$ using Fisher's exact test). The other was mean postoperative morphine requirement, which was noted to be higher in the retention group (34mg) versus those not developing retention (25mg) $(\mathrm{p}=0.035)$ (Table-1). No patients required urological surgical intervention at mean follow up of 1.97 years.

\section{COMMENTS}

Urinary retention and bladder overdistension may be missed surprisingly easily in the postoperative period especially with the altered sensorium that occurs with anesthetic and postoperative pain medications - the case of retention is thus "masked" as there is apparently little in the way of symptoms. The sequelae are not only limited to the immediate postoperative period because prolonged overdistension can lead to long-term bladder dysfunction. Once the bladder is stretched beyond its physical capacity then its ability to contract during voiding will be decreased. This overdistension causes ischemic injury to the bladder thus decreasing detrusor contractility and subsequent bladder decompression causes further injury. Decompression allows enhanced lipid peroxidation, which is associated with decreased metabolism thus impairing detrusor function even further (7). These processes are involved in the genesis of a "myogenic bladder" and the associated anesthesia and perioperative overhydration may exacerbate the condition further 
Table 1 - Demographics for all 142 patients divided into 3 groups: without catheter, preoperative catheter and postoperative catheter for acute urinary retention (AUR).

\begin{tabular}{lccc}
\hline & Without Catheter & Preoperative Catheter & Postoperative Catheter (AUR) \\
\hline Mean age & 72 & 74 & 73 \\
Total number & 97 & $19^{*}$ & 26 \\
M:F & $44: 53$ & $10: 9$ & $14: 12$ \\
GA:Spinal & $82: 15$ & $12: 7$ & $23: 3$ \\
Postoperation morphine - & & & 34 \\
$\quad$ mean in mg first 24 hours & 25 & 21 & - \\
Postoperation UTI & - & - & - \\
Joint sepsis & 2 & 1 & 5 \\
PMH LUTS & 9 & 2 & 4 \\
PMH AUR & 2 & 2 & 4 \\
PMH urological surgery & 5 & 1 & 4 \\
PMH alpha blockade & 1 & 1 &
\end{tabular}

Two patients with a preoperative catheter experienced retention after its removal. One was an eighty-one year old male who had spinal anesthesia with a PCA using $11 \mathrm{mg}$ of morphine. The other was an eighty-three year old woman who had a general anesthetic with $34 \mathrm{mg}$ postoperative morphine administered via a PCA. LUTS = lower urinary tract symptoms; AUR = acute urinary retention; $U T I=$ urinary tract infection; $G A=$ general anesthetic; $P M H=$ past medical history; $P C A=$ patient controlled analgesia .

with the length and degree of overdistension. Many of these patients will already have an aging bladder, which will not bear much further insult. In the effort to avoid catheterization, it is important therefore not to miss these patients with urinary retention and a high index of suspicion is advised especially if there is any previous history of lower urinary tract symptoms. A large bladder residual volume contributes to infection and the treatment is bladder drainage to avoid stagnation and therefore prevent urinary infection. There is a need to therefore identify those patients who are most at risk of urinary retention postoperatively so that they may be monitored more closely and catheterized early or even prophylactically before bladder overdistension occurs.

Deep joint sepsis following arthroplasty may occur by direct contamination of the wound or it may also arise from a distant site by haematogenous spread (8). The spread of infection from the urinary tract to cause deep joint sepsis has been well documented $(9,10)$. Deep joint sepsis is more common following knee arthroplasty with a quoted incidence of $2.5 \%$ as compared with $0.64 \%$ post hip arthroplasty (5). Septic arthritis of the knee is more difficult to treat than that involving the hip. The reasons postulated for this variance are multifold (6). The knee lies superficially and therefore has less well vascularized muscle covering. This may lead to poorer soft tissue healing. More of the knee is exposed to cement and there may be difficulty eradicating bacteria adherent to the cement. The reamed hole in the distal femur may also provide an escape pathway for bacteria after debridement.

It is because of the risks of joint sepsis that orthopedic surgeons remain concerned about urinary retention, urethral instrumentation and prolonged catheterization in the postoperative arthroplasty patient. Various differing approaches have been tried including indwelling (IDC) or intermittent catheterization (CIC) for all patients postoperatively, IDC or CIC for patients developing retention or alternatively to try to predict those at risk of acute urinary retention. IDC has been perceived to be associated with an increased incidence of urinary tract infection as opposed to CIC. Numerous studies have found no significant increased risk of urinary tract infection of IDC for 48 hours over CIC $(4,11,12)$. The study by Michelson et al. (12) however did note a significantly higher rate of infection if IDC was continued beyond 48 hours.

Our study identified previous urinary retention as a weak predictor $(\mathrm{p}=0.049)$ as a predisposing factor for subsequent retention. The evidence for 
postoperative morphine requirement predisposing to retention however was stronger $(\mathrm{p}=0.035)$. With our protocol, we observed a urinary retention rate of $19.7 \%$ (28/142). We kept the duration of catheterization to a minimum with catheter removal occurring as soon as the patient was mobile. The mean duration of catheterization in patients developing retention was 3.58 days and was 3 days in the patients catheterized pre - or perioperatively. According to our regimen, all patients received prophylactic cefuroxime as detailed above and where also given single doses of gentamicin with catheter insertion and removal. Deep joint sepsis occurred in $2.1 \%(3 / 142)$ - only one had been catheterized and that was preoperatively. No case of deep joint sepsis had urinary retention or had a symptomatic urinary tract infection.

\section{CONCLUSION}

This study offers a safe bladder management protocol post knee arthroplasty. The joint sepsis rates in this study are comparative for knee arthroplasty and none of these cases was associated with urinary retention or postoperative catheterization. The positive correlations between a previous history of retention and postoperative morphine requirements with the risk of postoperative retention allows for the closer monitoring of these patients postoperatively. This would allow for the prevention of bladder overdistension in these cases deemed at higher risk by the recognition of retention earlier on. These findings will therefore help to prevent the genesis of the "myogenic bladder" and its complications.

\section{CONFLICT OF INTERESTED}

None declared.

\section{REFERENCES}

1. Waterhouse N, Beaumont AR, Murray K, Staniforth $\mathrm{P}$, Stone MH: Urinary retention after total hip replacement. A prospective study. J Bone Joint Surg Br. 1987; 69: 64-66.

2. Oishi CS, Williams VJ, Hanson PB, Schneider JE,
Colwell CW Jr, Walker RH: Perioperative bladder management after primary total hip arthroplasty. $\mathrm{J}$ Arthroplasty. 1995; 10: 732-6.

3. Wroblewski BM, del Sel HJ: Urethral instrumentation and deep sepsis in total hip replacement. Clin Orthop Relat Res. 1980; 146: 209-12.

4. Iorio R, Healy WL, Patch DA, Appleby D: The role of bladder catheterization in total knee arthroplasty. Clin Orthop Relat Res. 2000; 380: 80-84.

5. Wymenga AB, van Horn JR, Theeuwes A, Muytjens HL, Slooff TJ: Perioperative factors associated with septic arthritis after arthroplasty. Prospective multicenter study of 362 knee and 2,651 hip operations. Acta Orthop Scand. 1992; 63: 665-71.

6. Segawa H, Tsukayama DT, Kyle RF, Becker DA, Gustilo RB: Infection after total knee arthroplasty. A retrospective study of the treatment of eighty-one infections. J Bone Joint Surg Am. 1999; 81: 1434-45.

7. Lin AT, Chen KK, Yang CH, Chang LS. Mannitol facilitates rabbit urinary bladder recovery from overdistension injury. Urology. 2000; 56: 702-7.

8. Ahlberg A, Carlsson AS, Lindberg L. Hematogenous infection in total joint replacement. Clin Orthop Relat Res. 1978; 137: 69-75.

9. Irvine R, Johnson BL Jr, Amstutz HC: The relationship of genitourinary tract procedures and deep sepsis after total hip replacements. Surg Gynecol Obstet. 1974; 139: 701-6.

10. Donovan TL, Gordon RO, Nagel DA: Urinary infections in total hip arthroplasty. Influences of prophylactic cephalosporins and catheterization. $\mathbf{J}$ Bone Joint Surg Am. 1976; 58: 1134-7.

11. Ritter MA, Faris PM, Keating EM: Urinary tract catheterization protocols following total joint arthroplasty. Orthopedics. 1989; 12: 1085-7.

12. Michelson JD, Lotke PA, Steinberg ME: Urinarybladder management after total joint-replacement surgery. N Engl J Med. 1988; 319: 321-6.

Accepted after revision:

September 30, 2005 